Midwives' experiences of traumatic perinatal events

1 TITLE: EXPOSURE TO TRAUMATIC PERINATAL EXPERIENCES AND

2 POSTTRAUMATIC STRESS SYMPTOMS IN MIDWIVES: PREVALENCE AND

3 ASSOCIATION WITH BURNOUT

4 RUNNING HEAD: MIDWIVES EXPERIENCES OF TRAUMATIC PERINATAL

\title{
5 EVENTS
}

6

7

8
Dr Kayleigh Sheen

University of Liverpool, UK

Professor Helen Spiby

University of Nottingham, UK

Professor Pauline Slade

University of Liverpool, UK

\section{Author Note:}

Dr Kayleigh Sheen, Institute of Psychology, Health and Society, University of Liverpool, Liverpool, UK; Professor Helen Spiby, School of Health Sciences, University of Nottingham, Nottingham, UK; Pauline Slade, Institute of Psychology, Health and Society, University of Liverpool, Liverpool, UK. The authors would like to thank the midwives who provided their time to share their experiences within this survey, and to those who aided in the development and piloting of the study. The authors also thank the Royal College of Midwives' for supporting the sampling and distribution strategy.

3 Please address correspondence to: Dr Kayleigh Sheen, Psychological Sciences,

24 Institute of Psychology, Health and Society, University of Liverpool, Liverpool, L69 3GB.

Email: Kayleigh.sheen@liverpool.ac.uk 
Midwives' experiences of traumatic perinatal events

1 ABSTRACT: Background: Midwives provide care in a context where life threatening or

2 stressful events can occur. Little is known about their experiences of traumatic events or the

3 implications for psychological health of this workforce. Objectives: To investigate

4 midwives' experiences of traumatic perinatal events encountered whilst providing care to

5 women, and to consider potential implications. Design: A national postal survey of UK

6 midwives was conducted. Participants: 421 midwives with experience of a perinatal event

7 involving a perceived risk to the mother or baby which elicited feelings of fear, helplessness

8 or horror (in the midwife) completed scales assessing posttraumatic stress (PTS) symptoms,

9 worldview beliefs and burnout. Results: $33 \%$ of midwives within this sample were

10 experiencing symptoms commensurate with clinical posttraumatic stress disorder (PTSD).

11 Empathy and previous trauma exposure (personal and whilst providing care to women) were associated with more severe PTS responses. However, predictive utility was limited, indicating a need to consider additional aspects increasing vulnerability. Symptoms of PTS were associated with negative worldview beliefs and two domains of burnout. Conclusions: Midwives may experience aspects of their work as traumatic and, as a consequence,

16 experience PTS symptomatology at clinical levels. This holds important implications for both midwives' personal and professional wellbeing and the wellbeing of the workforce, in addition to other maternity professionals with similar roles and responsibilities. midwives following traumatic perinatal events and provide effective intervention for those 21 with significant symptoms.

23 KEY WORDS: Burnout; midwives; trauma; posttraumatic stress 
Midwives' experiences of traumatic perinatal events

\section{MAIN PAPER}

\section{Introduction}

3 Childbirth is generally considered in the developed world to be a normal, positive event.

4 However adverse events can occur during the perinatal period, whereby the mother or her

5 child is at risk of death or serious injury. Instances such as these can fulfil criteria for a

6 traumatic event (APA, 2013). A proportion of both mothers and fathers perceive their

7 experience of or being present at childbirth to be traumatic (Czarnocka \& Slade 2000,

8 Bradley et al., 2008), but there is a paucity of research considering midwives' perceptions of

9 such events (Sheen, Slade \& Spiby, 2014).

A traumatic birth is considered to be an event involving actual or threatened serious injury or death to the mother or her child (APA, 2013; Beck, 2004). There is potential for midwives to indirectly experience traumatic perinatal events either by witnessing them or by listening to accounts of birthing episodes from women, both of which are encompassed within the definition of trauma exposure (APA, 2013). Indirect exposure to trauma has been associated with a number of adverse psychological responses, including posttraumatic stress 16 disorder (PTSD).

PTSD is defined by the American Psychological Association (APA) in the Diagnostic and Statistical Manual of Mental Disorders (DSM-V; APA, 2013), which is internationally endorsed by organisations including the Royal College of Psychiatrists and the Australian Centre for Posttraumatic Mental Health. Trauma exposure (referred to as Criterion A) is

21 defined as exposure to an event involving perceived threat to the self or somebody else's life

22 (APA, 2013). The predecessor to the DSM-V (the DSM-IV; APA, 2000) included requirement for appraisal of the event to involve fear, helplessness or horror; however, this

24 was removed from the diagnostic criteria in the newest version. This study uses the definition 25 of trauma exposure from the DSM-IV to account for both trauma exposure and appraisal. 
Midwives' experiences of traumatic perinatal events

PTSD is characterised by symptoms of intrusion (distressing, involuntary recollections), avoidance (of reminders, thoughts of the event) and increased arousal. The fourth dimension relates to the potential for the development of negative emotions (fear, guilt, shame) and exaggerated, negative worldview beliefs (APA, 2013). Exposure to trauma through providing care has the potential to elicit symptomatic responses of posttraumatic stress (PTS) (Elwood et al., 2011) and PTS symptoms have been reported by a variety of health professional groups including nurses (Mealer et al., 2012) and ambulance drivers (Alexander \& Klein, 2001).

Burnout is often implicated in studies assessing responses to trauma in health professionals, characterised by high levels of emotional exhaustion, the distancing of oneself from recipients of care through depersonalisation, and reduced personal accomplishment (Maslach et al., 1996). It is not a response to trauma, but a response to chronic strain in the workplace; however, symptoms of burnout have been identified as highly associated with (yet distinct from) symptomatic responses to trauma (Jenkins \& Baird, 2005). Burnout has been associated with high levels of staff turnover and absenteeism (Leiter \& Maslach, 2009), with important implications for professional wellbeing and organisational efficiency.

The International Confederation of Midwives (ICM) defines a midwife as a responsible and accountable professional, who works in partnership with women to support and provide care (ICM, 2011). It is important to understand aspects of midwifery practice that may hold adverse implications for midwives' psychological health, and which may subsequently impact upon capacity to provide sensitive maternity care. Sheen, Slade and Spiby (2014) note the limited research investigating the potential for traumatic childbirthrelated events to elicit symptoms of traumatic stress in attending professionals. A study with UK midwives and obstetricians identified symptoms of intrusion and avoidance after encountering miscarriage, stillbirth and neonatal death (Wallbank, 2010). However the 
Midwives' experiences of traumatic perinatal events

1 proportion of midwives in this sample was small and it was impossible to disaggregate

2 midwives' symptomatic responses from those of obstetricians'. Beck and Gable (2012)

3 reported that $35 \%$ of their sample of labour and delivery nurses in America experienced

4 moderate to severe symptoms synonymous with PTS. Their large sample and national

5 recruitment strengthen the extent to which findings from that study can be extrapolated.

6 The majority of studies to date have been conducted with nurses specialised in the

7 provision of intrapartum care (e.g., Beck \& Gable, 2012; Beck \& Gable, 2013; Goldbort et

8 al., 2011). Midwives in the UK are autonomous, independent practitioners who are able to

9 provide all aspects of maternity care to women considered at low risk (Department of Health,

10 2010). Due to differences in role autonomy between these different contexts (Mallot et al.,

11 2009), large-scale research specifically considering midwives' experiences is required.

12 Several aspects identified as increasing vulnerability to traumatic stress responses in

13 other health professionals hold salience for midwives (Sheen, Slade \& Spiby, 2014),

14 including empathic engagement with recipients of care and working in a stressful

15 environment. Empathic engagement with women is fundamental in maternity care

16 (Department of Health, 2010) and is a highly valued aspect of midwifery practice (Thomas,

17 2006). However, it is recognised to increase vulnerability to traumatic stress responses

18 (Figley, 1995). In addition, midwifery in the UK can be highly stressful (Birch, 2001).

19 In terms of other potential predictors, duration of a professional's experience has also

20 been identified as associated with subsequent traumatic stress responses (Beck \& Gable,

21 2012). However the direction of association is not consistently identified (Sheen, Slade \&

22 Spiby, 2014). Personal (and direct) experiences of trauma could also predispose to traumatic

23 stress responses following subsequent (indirect) exposure (Breslau et al., 2009). Personal

24 experience of childbirth trauma could be specifically salient for midwives (e.g., Mander, $252001)$. 
Midwives' experiences of traumatic perinatal events

There is potential for midwives to indirectly experience events in their professional

2 role, which may fulfil criteria for trauma. However there is a need to identify the proportion

3 of midwives experiencing and responding to traumatic perinatal events and to assess the

4 utility of factors that may identify those most vulnerable. It is also important to explore any

5 association between traumatic stress responses and burnout, to identify the potential

6 implications for professional and personal wellbeing.

The aim of this study was to investigate the psychological impact of exposure to

8 traumatic perinatal events in midwives. Specifically, this study aimed to report the proportion

9 of midwives experiencing traumatic perinatal events and the nature of impact these had,

10 including impacts to professional lives and prevalence of PTS symptomatology. The

11 association between aspects highlighted as increasing vulnerability (empathy, personal

12 trauma history, extent of experience in the profession, extent of exposure to traumatic events)

13 and PTS symptoms was also investigated. Finally, associations between PTS symptoms and

14 burnout were investigated.

15

\section{METHOD}

\section{Design}

18 A postal survey was conducted.

\section{Ethical Approval}

21 The Department of Psychology Ethics Committee, University of Sheffield provided ethical 22 approval.

\section{Participants}


Midwives' experiences of traumatic perinatal events

1 Participants were qualified midwives, currently employed in the United Kingdom. Experience

2 of a traumatic perinatal event was defined Criterion A of the DSM-IV-TR (APA, 2000); that

3 the midwife witnessed or listened to an account of an event where they perceived the mother

4 and/or her child to be at risk of serious injury or death and where they (the midwife)

5 experienced a sense of fear, helplessness or horror. Postal surveys were distributed to 2800

6 midwives, randomly selected from the Royal College of Midwives' (RCM) membership

7 database between December 2011 and April 2012. The RCM is the UK's largest trade union

8 and professional organisation for midwives and the majority of midwives within the UK are

9 members. At the time of conducting the study the approximate number of members, as

10 estimated by an RCM representative, was 30,000. Qualitative descriptions and perceived

11 implications of traumatic perinatal event experiences were collated and are presented 12 separately to this manuscript.

\section{Measures}

15 The postal survey was developed through liaison with a reference group of expert midwives.

16 Midwives' age, gender, marital status, ethnicity, highest midwifery-related qualification,

17 parity were obtained, together with years' experience in the profession, professional

18 designation and current job role, current clinical activity, employer and NHS Band (if

19 applicable). Personal trauma experience was assessed using criterion A of the DSM-IV-TR

20 (APA, 2010) for PTSD. Midwives were also asked whether they considered their own

21 personal experience of giving birth (if applicable) to be traumatic.

22 Midwives were asked to estimate the number of events that met the criteria that they

23 had experienced over the full duration of their career. Midwives indicated whether they had

24 1) ever taken time off sick, 2) changed their professional allocation on short or long-term 
Midwives' experiences of traumatic perinatal events

1 basis, and 3) whether they had ever seriously considered leaving the midwifery profession

2 due to a traumatic perinatal event experience.

The primary outcome measure was the severity of posttraumatic stress

symptomatology, measured using the Impact of Event Scale- Revised (IES-R, Weiss \&

Marmer, 1997). There are 22 items and three subscales, measuring symptoms of intrusion,

avoidance and arousal. Responses are scored based on the degree of current difficulty on a scale of 0 (not at all) to 4 (extremely); therefore total scores range in from $0-88$. The IES-R has demonstrated excellent internal validity (Weiss and Marmer, 1997). Whilst the IES-R is not a diagnostic measure for PTSD, a total of $\geq 34$ has been reported to predict clinical diagnosis of PTSD with sensitivity of $70 \%$, specificity of $77 \%$, positive predictive value of 0.81 and negative predictive value of 0.66 (Rash, 2008). This cut off was applied to total scores on the IES-R $\mathrm{n}$ this study to infer presence of PTS at levels indicative of clinical relevance. Assumptions Scale (WAS, Janoff-Bulman, 1989). The WAS consists of 32 items measuring beliefs about the benevolence of the world, meaningfulness of the world and self-worth.

17 Responses are scored in terms of the degree of endorsement from 1 (strongly disagree) to 6 (strongly agree); higher scores indicate more positive worldview beliefs. Scores on the WAS can therefore range from 32-192. Janoff-Bulman (1989) reported good levels of internal consistency for each subscale.

22 Services Survey (MBI, Maslach et al., 1996), which is designed to specifically assess 23 responses in individuals engaged in a capacity of care. The MBI measures emotional 24 exhaustion (EE; 9 items), depersonalisation (DP; 5 items) and personal accomplishment (PA; 258 items) using three subscales and yields three scores for each. Responses are scored in terms 
Midwives' experiences of traumatic perinatal events

1 of the frequency at which each item is experienced on a scale of 0 (never) to 6 (every day).

2 Scores for EE can range from 0-54, DP 0-30 and PA 0-48. Maslach et al. (1996) reported

3 good internal consistency for each subscale of the MBI. Higher scores on the EE and DP and

4 lower scores on the PA scale indicate more severe burnout. Scores in the upper third of the

5 normative distribution are indicative of 'high' levels of burnout, 'moderate' if they are in the

6 middle third and 'low' if they are in the lower third (Maslach et al., 1986). Several items in

7 the scale referred to 'recipients' of care. To minimise confusion, this was replaced with

8 'women in my care' for the present study.

Empathy was measured using the Empathic Concern (EC) subscale from the

Interpersonal Reactivity Index (IRI; Davis, 1983). There are 7 items for this subscale, scored

11 on a scale of 1-5 thus producing scores with a potential range of 7- 35. The IRI has

12 demonstrated good internal consistency (Davis, 1980).

\section{Data analysis}

15 Statistical analyses were conducted using IBM SPSS 19. Descriptive statistics were computed

16 for total scores on the IES-R, MBI, WAS and IRI. Pearson product moment correlation coefficients were conducted to assess associations between scores on the IES-R and MBI, and IES-R and WAS. Standard multiple regression analysis was used to investigate the association between variables identified as associated with PTS symptoms (empathy, number and extent of exposure to traumatic perinatal event experiences, personal trauma history).

21 Standard multiple regression analysis was used to further assess the association between scores on the IES-R and WAS for each subscale on the MBI. A $p$ value of $\geq .05$ was considered statistically significant.

\section{RESULTS}


Midwives' experiences of traumatic perinatal events

\section{Sample characteristics}

2 Four hundred and sixty four questionnaires were returned (16\% response rate). Of these

3 midwives, 421 had experienced at least one traumatic perinatal event and thus formed the

4 final sample for analysis. Descriptive statistics for midwives in this sample are presented in

5 Table 1. Midwives had been qualified between six months to 44 years $(M=17.28, S D=$

6 10.48). The majority of midwives in this sample $(n=397,94 \%)$ were employed in a National

7 Health Service (NHS) Trust, a healthcare system providing NHS maternity care which is free

8 at point-of-service. Respondents reported employment at Bands 5-8d, with the majority

9 employed at Band 6 level $(n=272,65 \%)$. Ninety five percent of respondents were engaged in

10 clinical practice on at least a monthly basis $(n=395,94 \%)$ at the time of the survey. The most

11 frequently reported area for current practice was care during labour and birth $(n=253,60 \%)$.

\section{Experiences of traumatic perinatal events}

14 Midwives had experienced an average of 7 traumatic perinatal events $(M=6.63, S D=9.93)$

15 throughout their career. The number of traumatic perinatal events experienced throughout

16 midwives' careers was used as an indication of total exposure to traumatic perinatal events.

17 Nearly all midwives had witnessed a traumatic perinatal event $(n=402,95 \%)$ and nearly three

18 quarters of the sample $(n=318,76 \%$ ) had listened to an account of an event from a woman in

19 their care that they (the midwife) perceived to be traumatic. The majority of the sample had

20 both witnessed and listened to accounts of events perceived to be traumatic $(n=299,71 \%)$,

21 however a smaller proportion of the sample had only witnessed ( $n=103,24 \%)$ or only

22 listened to accounts of events perceived as traumatic $(n=19,5 \%)$. The degree of exposure to

23 traumatic perinatal events was subsequently coded to indicate both types of exposure

24 (witnessed and listened to accounts), or only one type of exposure (just witnessed, or just

25 listened to accounts). 
Midwives' experiences of traumatic perinatal events

\section{Perceived impact of traumatic perinatal event exposure}

3 A third had seriously considering leaving the midwifery profession after experiencing a

4 traumatic perinatal event $(n=148,35 \%)$. A fifth $(n=82,20 \%)$ changed their professional

5 allocation on a short-term basis (e.g., for a small number of shifts) after experiencing a

6 traumatic perinatal event. For a smaller proportion, a long-term (or permanent) change in

7 professional allocation was sought $(n=57,14 \%)$. Just over $10 \%$ of this sample had taken time

8 away from employment after experiencing a traumatic perinatal event $(n=50,12 \%)$.

9 Mean PTS score for midwives who had seriously considered leaving the midwifery

10 profession were significantly higher than those reported by midwives who had not considered

11 leaving the profession following a traumatic perinatal event, $t(412)=10.4, p<.001$.

13 Posttraumatic stress symptoms (PTS)

14 Midwives' mean total and factor scores on the IES-R are shown in Table 2. Cronbach's alpha

15 coefficients for intrusion, avoidance and arousal subscales were indicative of good internal

16 consistency $(90, .82$ and .87 respectively). Approximately one third of midwives who

17 participated $(n=138,32 \%)$ exceeded the cut off score indicative of symptoms commensurate

18 with a clinical diagnosis of PTS.

19 Midwives' mean scores across all subscales of the WAS are shown in Table 2. Alpha

20 coefficients for the benevolence of the world, and self-worth subscales were indicative of

21 good internal consistency within this study (both .79). However the coefficient for

22 meaningfulness of the world was .69, slightly below the level considered acceptable

23 (Nunnally \& Bernstein, 1994). There was a small, significant association between higher

24 scores on the IES-R and lower scores on the WAS, $r(411)=-.238, p<.001$, indicating that

25 higher symptoms of PTS were associated with more negative worldview schema.. 
Midwives' experiences of traumatic perinatal events

\section{Factors associated with PTS}

3 Bivariate correlations (Pearson's $r$ ) were conducted to assess linearity between midwives'

4 total PTS score and their length of experience in the profession, total number of traumatic

5 perinatal experiences and their extent of exposure to these (witnessed and heard, just

6 witnessed or heard), personal trauma history (general and childbirth related considered

7 separately), and empathy (see Table 3). For the empathic concern subscale of the IRI,

8 Cronbach's alpha coefficient for the present study was acceptable (.70).

There were small, significant associations between total IES-R score and total number

10 of traumatic experiences $(r=.181, p=.001)$, extent of exposure to traumatic perinatal events

$11(r=.212, p<.001)$, having a personal trauma history $(r=-.119, p=.034)$ and higher empathy

$12(r=.129, p=.021)$. Total IES-R score was not significantly associated with midwives'

13 experience in the profession $(r=.037, p=.509)$ or, where applicable, whether the midwife had

14 personally experienced a traumatic childbirth $(r=.011, p=.839)$.

Variables that were significantly associated with total score on the IES-R were entered

16 into a standard multiple regression to further investigate these associations. There was evidence of positive skew on all subscales of the IES-R, and negative skew on the empathic concern subscale of the IES-R. This was improved through square-root transformation (reflected where negative skew was present), however comparison of analysis on transformed and un-transformed data indicated no difference in the significance of analysis and minimal

21 difference in the magnitude of associations. As the total sample was large ( $\mathrm{n}=421)$, and parametric tests are considered more robust to violations of normality when conducted on larger sample sizes, analysis is presented using non-transformed data. Collinearity diagnostics

24 and scatterplot diagrams were inspected for evidence of multicollinearity and histogram and 
Midwives' experiences of traumatic perinatal events

1 that model parameters adequately conformed to the assumptions of multiple regression

2 analysis.

Total exposure to traumatic perinatal events, the extent of exposure (whether events were witnessed or listened to), total score for empathy, personal trauma history (general, childbirth) were entered as independent variables (see Table 4). The regression was significant $(F(4,320)=6.388, p<.001)$, but the combination of variables accounted for only $6 \%$ of variance in PTS symptoms.

\section{Associations between PTS, worldview beliefs and burnout}

Descriptive statistics and category interpretations for severity of scores on the MBI are presented in Table 2. Alpha coefficients for subscales on the MBI for the present study were .90 (EE), .69 (DP) and .73 (PA), indicating excellent (EE) and acceptable (PA) internal consistency, with the DP subscale showing internal consistency just short of the level considered acceptable.

A higher level of overall PTS symptomatology was moderately associated with a higher emotional exhaustion $(r(385)=.420, p<.001)$. There was a small association between more severe symptoms of overall PTS and a greater level of depersonalisation $(r(385)=.247$, $p<.001)$. There was no significant association between overall PTS symptomatology and perceptions of personal accomplishment $(r(385)=-.018, p=.339)$. More negative worldview schema were associated with a higher levels of emotional exhaustion $(r(386)=-.311, p<.001)$ and depersonalisation $(r(386)=-.259, p<.001)$. More positive worldview beliefs were associated with a greater level of perceived personal accomplishment $(r(386)=.263, p<.001)$. Findings from bivariate correlations are presented in Table 5.

To further assess the association between traumatic-stress responses (PTS and worldview beliefs) and burnout responses, standard multiple regression analyses were 
Midwives' experiences of traumatic perinatal events

1 conducted with the linearly related subscales of posttraumatic stress (intrusion, avoidance and

2 arousal) and worldview schema (benevolence of the world, self-worth) as independent

3 variables and the level of emotional exhaustion, depersonalisation and personal

4 accomplishment as DV's (see Table 6). The benevolence of the world and self-worth

5 subscales of the WAS were negatively skewed. Similar to the IES-R subscales, the emotional

6 exhaustion and depersonalisation subscales of the MBI were positively skewed. As with the

7 previous regression analysis, transformations had little impact on the outcome of analysis and

8 results are presented from the analysis of the untransformed data. Model parameters were

9 checked and adequately conformed to the assumptions of multiple regression analysis.

The regression model for emotional exhaustion (EE) was significant, $F(5,378)=$

$1124.240, p<.001$, and the combined symptoms of PTS and worldview beliefs accounted for

$1223 \%$ of the variance in EE. Avoidant symptomatology $(t(378), 2.690, p=.007)$, beliefs about

13 the benevolence of the world $(t(378)=-.1997, p=.047)$ and beliefs about self-worth $(t(378)=-$

$144.149, p<.001)$ were uniquely associated with PTS. The regression model for

15 depersonalisation (DP) was significant, $F(5,378)=12.927, p<.001$, and accounted for $14 \%$ of

16 the variance. Avoidance $(t(377)=2.800, p=.005)$, benevolence of the world $(t(377)=-4.194$,

$17 p<.001)$ and self-worth $(t(377)=-2.112, p=.035)$ were uniquely associated with DP.

18 Only worldview beliefs about the benevolence of the world and self-worth were

19 linearly associated with personal accomplishment, and therefore only these were entered into

20 a standard multiple regression analysis. This was significant, $F(2,385)=23.661, p<.001$, and

21 accounted for $11 \%$ of variance in perceived personal accomplishment. Both regression

22 coefficients were uniquely associated with personal accomplishment; beliefs about the

23 benevolence of the world $(t(385)=2.950, p=.003)$ and self-worth $(t(377)=4.468, p<.001)$. 
Midwives' experiences of traumatic perinatal events

1 This is the first large UK based survey of midwives' experiences of traumatic events. A third

2 of the sample reported current posttraumatic stress symptoms at levels indicative of clinical

3 relevance following exposure to a traumatic perinatal event. Symptoms of posttraumatic

4 stress were associated with more negative worldview beliefs and higher levels of burnout in

5 the form of emotional exhaustion, and depersonalisation.

6 The age and gender ratio of the current sample was similar to qualified midwives in

7 the UK (NMC, 2008); the majority of midwives in England are female (99.6\%), and the

8 largest proportion of midwives (approximately 20\%) are aged between 40-45 years (RCM,

9 2013) and 50-54 years in Scotland (ISD Scotland, 2014). The proportion of midwives in this

10 sample with a personal trauma history, general or specific to childbirth, was lower than

11 percentages reported within community samples (HSCIC, 2009) and in previous research

12 with mothers in the UK (Olde et al., 2006).

13 The sampling strategy employed within this study provides a conservative estimation

14 of the minimum proportion of midwives in the UK likely to be experiencing clinically

15 relevant symptoms of PTS. By assuming that midwives not returning the questionnaire were

16 entirely non-symptomatic (85\%), and the $15 \%$ who returned the questionnaire represent

17 midwives experiencing difficulty following a traumatic perinatal event, it can be estimated

18 that approximately one third of this $15 \%$ (or a minimum of $5 \%$ of the midwifery profession)

19 may at some point experience symptoms of PTS commensurate with a clinical diagnosis. This

20 will underestimate the proportion of midwives experiencing symptoms, as it is highly likely

21 that a proportion of midwives will not have returned the questionnaire due to avoidance,

22 which is in itself an element of PTS symptomatology. Furthermore this estimation does not

23 take into account midwives with sub-threshold, but highly distressing symptoms.

24 Few studies have assessed PTS in healthcare professionals using the IES-R. Wallbank

25 (2010), as part of a pilot study, assessed symptoms of intrusion and avoidance in a small 
Midwives' experiences of traumatic perinatal events

1 sample of UK midwives and obstetricians $(n=30)$ using the IES. The IES is the predecessor to

2 the IES-R, and assesses intrusion and avoidance factors only. However, scores for these two

3 subscales are comparable to scores from the same subscales included in the IES-R (Horowitz,

4 Wilner \& Alvarez, 1979). Wallbank (2010) reported similar levels of intrusion $(M=12.06$,

$5 S D=8.31)$ and avoidance $(M=10.20, S D=7.61)$ within their sample, to those reported in the

6 current sample.

Midwives reporting a higher number of traumatic perinatal events, who had

8 experienced these through both types of exposure (i.e., witnessed and listened to), had

9 personally experienced trauma and were more empathic reported more severe symptoms of

10 PTS. Particularly associated were the frequency and extent of perceived event exposure,

11 indicative of a cumulative symptomatic response. However the amount of variance explained within this model was low. These variables were originally selected for investigation due to their pertinence in the wider trauma literature (Sheen, Slade \& Spiby, 2014). However,

14 findings indicate that the predictive utility of these aspects was limited for this midwifery sample. Further investigation and consideration of additional aspects increasing vulnerability to traumatic stress responses within midwives is required. corresponds to the revised symptom profile of PTSD in the DSM-V (APA, 2013). The relationship between worldview beliefs and PTS symptomatology is potentially bidirectional 20 (Dekel, Paleg \& Solomon, 2013). It could be that midwives' beliefs about the self, world and others people were contradicted by the occurrence of a traumatic perinatal event, which contributed to the extent of disruption and symptomatic responses afterwards (Ehlers \&

23 Clark, 2000). However it could also be that midwives' perceptions of the self, world and

24 other people were negative prior to the event and the occurrence of a traumatic perinatal event provided confirmatory evidence (Foa \& Rothbaum, 1989). Without implementation of 
Midwives' experiences of traumatic perinatal events

1 a prospective research design, the contribution of cognitive schema for the development of

2 PTS response is unclear.

In comparison to normative values for burnout (Maslach et al., 1996), midwives

4 reported generally high emotional exhaustion, low depersonalisation and low personal

5 accomplishment. Respondents also reported a greater level of emotional exhaustion, less

6 perceived personal accomplishment and were exhibiting greater depersonalisation toward

7 women in their care in comparison to other studies with midwives (Bakker et al., 1996;

8 Mollart et al., 2013). Bakker et al. (1996) investigated burnout in Dutch community

9 midwives, and therefore differences between these respondents and midwives in the present

10 sample (e.g., high professional status in Holland, the majority of midwives in the UK sample

11 worked in hospital settings) may contribute to different levels of burnout.

Forty percent of respondents in the present study reported high levels of emotional

exhaustion, and four percent reported a high level of depersonalisation towards recipients of

care. Emotionally exhausted midwives are less likely to be able to engage in compassionate

care. Mollart et al's (2013) study with Australian midwives also identified a potential association between increased burnout and a reduction of empathic care for women. The importance of compassion in maternity care is a key priority in England, as stipulated in the Department of Health's 6 C's (DOH, 2012), and on a global basis (ten-Hoope-Bender et al., 2014). Elmir et al. (2010) reported that mothers who felt disregarded by professionals whilst giving birth were more likely to perceive childbirth as traumatic. Therefore midwives' experiences of traumatic perinatal events, and their subsequent responses to these, hold important implications for the experiences of mothers receiving care and the efficiency of maternity services.

The association between empathy and PTS responses also indicates that highly 
Midwives' experiences of traumatic perinatal events

1 normed values (Davis, 1980), midwives reported a greater level of empathic concern.

2 However, it could be argued that the nature of midwifery care may mean that high levels of

3 empathic concern are to be expected. Given the additional association identified between

4 PTS symptomatology and considerations over leaving the profession, it is possible that the

5 midwifery profession may risk losing the most empathic midwives following traumatic

6 perinatal event exposure. Furthermore a proportion of midwives in this sample took time

7 away from practice or changed their clinical allocation after a traumatic perinatal experience.

8 High levels of exhaustion, increased absenteeism and attrition from the workforce are likely

9 to increase existing constraints on UK maternity services.

\section{Implications and recommendations}

12 Conservative estimations suggest that at least $5 \%$ of UK midwives are experiencing symptoms commensurate with PTSD, and that the severity of these symptoms are highly associated with increased burnout. It must be noted that this represents the absolute minimum prevalence, as it is highly likely that a proportion of midwives did not return the questionnaire due to avoidance or the associated distress of recounting their experiences. This percentage also does not take into account midwives with sub-threshold symptoms, who are also likely to experience distress.

To contextualise these findings; of the 25,654 midwives registered to practice within England (HSCIC, 2013), over four thousand midwives $(n=4104)$ could experience traumatic 21 perinatal events through providing care to women, and almost thirteen hundred $(n=1282)$ could be experiencing PTS responses. Responses of PTS were highly associated with two domains of burnout. With rising birth rates throughout most of the UK and a high proportion

24 of midwives within ten years of retirement (RCM, 2013), there is a fundamental need to 25 acknowledge aspects of practice that impact upon professional wellbeing. 
Midwives' experiences of traumatic perinatal events

Midwives in this sample were predominantly employed in a healthcare organisation (an NHS Trust). Trusts employing midwives hold a duty of care for employees to support their wellbeing (DOH, 2011). It is essential that midwifery services acknowledge the potential for some midwives to experience events encountered at work as traumatic, for some to experience PTS-type responses, and that the development of strategies to support midwives is required.

\section{Findings also hold implications for midwifery practice internationally where the ICM} definition of midwifery applies, and for other maternity professionals who may experience similar events through their clinical practice. This study contributes to the emerging international interest in maternity professionals' experiences of adverse obstetric events (e.g., Beck \& Gable, 2012). A shortage of midwifery staff has been reported in contexts other than the UK, such as Australia (AHMC, 2011; Health Workforce Australia, 2012). Avoiding attrition from the midwifery workforce and supporting midwives in their working environments are important ways of ensuring that the care provided to women is compassionate and of high quality, which is of global importance (ten Hoope-Bender et al., 2014).

\section{Strengths and limitations}

The large sample size for the postal survey provided sufficient power with which to conduct statistical tests. The national and random nature of the sampling strategy provided a sample of midwives whose demographic profile reflected that of midwives within the UK. The $16 \%$ response rate of this sample indicates that extrapolation of findings to the general midwifery populations must be conservatively drawn; however, similar response rates are reported in studies with other healthcare professionals. Due to the recruitment strategy used details of non-responders could not be obtained, and so there is potential for selection bias. Alpha 
Midwives' experiences of traumatic perinatal events

1 coefficients for most subscale scores were indicative of acceptable internal consistency

2 (Nunnally \& Bernstein, 1994). Alpha coefficients for the MW subscale of the WAS and the

3 DP subscale of the MBI were slightly lower (.65 and.69 respectively). Whilst this can be

4 considered acceptable given the diversity of the construct being measured (Kline, 1999),

5 findings should be interpreted conservatively. Significant associations were identified

6 between symptoms of PTSD and empathy, number and extent of exposure to traumatic

7 perinatal event experiences, presence of a personal trauma history, but the magnitude of the

8 associations were small. The retrospective and cross-sectional design of this study enabled

9 efficient collection of a large body of data; however, to establish causal relationships

10 prospective and longitudinal research designs are essential.

11

\section{Conclusions}

13 This is the first large-scale investigation into UK midwives' experiences and responses to

14 traumatic perinatal events. Findings from this study indicate that midwives experience some

15 events they encounter in their practice as traumatic, and a significant subsample are

16 experiencing clinically relevant PTS symptomatology. A higher number of previous

17 traumatic event experiences, and a greater degree of exposure to these increased the risk of

18 developing higher symptoms of posttraumatic stress symptomatology, however the predictive

19 utility of these variables was limited. Symptoms of PTS were associated with elevated

20 symptoms of burnout, namely emotional exhaustion and reflected the depersonalisation of

21 recipients of care. There is potential for implications to extend to the experiences of women

22 in receipt of maternity care and for the efficiency of maternity services. Subsequent research

23 addressing effective ways of preparing and supporting midwives following traumatic

24 perinatal events is essential. 
Midwives' experiences of traumatic perinatal events

\section{REFERENCES}

2 Australian Health Ministers' Conference. (2011). National Maternity Services Plan 2010 (D0159). Commonwealth of Australia.

Alexander, D. A., \& Klein, S. (2001). Ambulance personnel and critical incidents - Impact of accident and emergency work on mental health and emotional well-being. British Journal of Psychiatry, 178, 76-81. doi: 10.1192/bjp.178.1.76

American Psychological Association. (2000). Diagnostic and Statistical Manual of Mental Disorders (IV-TR). Washington DC: American Psychiatric Association.

American Psychological Association. (2013). Diagnostic and Statistical Manual of Mental Disorders (V). Washington DC: American Psychiatric Association.

Bakker, R. H. C., Groenewegen, P. P., Jabaaij, L., Meijer, W., Sixma, H., \& deVeer, A. (1996). 'Burnout' among Dutch midwives. Midwifery, 12(4), 174-181. doi:

\section{$10.1016 / \mathrm{s} 0266-6138(96) 80004-0$}

Beck, C. T. (2004). Birth trauma: In the eye of the beholder. Nursing Research, 53(1), 28-35.

Beck, C. T., Gable, R. K. (2012). A mixed methods study of secondary traumatic stress in labour and delivery ward nurses. Journal of Obstetric Gynecological Neonatal Nursing, 41(6), 747- 760. doi: 10.1111/j.1552-6909.2012.01386.x

Birch, L. (2001). Stress in midwifery practice: an empirical study. British Journal of Midwifery, 9(12), 730-734.

Bradley, R., \& Slade, P., \& Leviston, A. (2008). Low rates of PTSD in men attending childbirth: a preliminary study. British Journal of Clinical Psychology, 47(3), 295302. doi: 10.1348/014466508X279495

Breslau, N. (2009). The epidemiology of trauma, PTSD, and other posttrauma disorders. Trauma Violence \& Abuse, 10(3), 198-210. doi: 10.1177/1524838009334448 
Midwives' experiences of traumatic perinatal events

1 Czarnocka, J., \& Slade, P. (2000). Prevalence and predictors of post-traumatic stress symptoms following childbirth. British Journal of Clinical Psychology, 39, 35-51. doi: 10.1348/014466500163095

Davis, M.H. (1980). A multidimensional approach to individual differences in empathy. JSAS Catalog of Selected Documents in Psychology, 10, 85- 104.

Davis, M. H. (1983). Measuring individual differences in empathy: Evidence for a multidimensional approach. Journal of Personality and Social Psychology, 44(1), 113-126.

Dekel, S., Peleg, T. \& Solomon, Z. (2013). The relationship of PTSD to negative cognitions: A 17-year longitudinal study. Psychiatry, 76(3), 241- 255. doi: 10.1521/psyc.2013.76.3.241

Department of Health. (2010). Midwifery 2020: Delivering expectations. London: Department of Health.

Department of Health. (2011). NHS Health and Well-being Improvement Framework. London: Department of health.

Ehlers, A., \& Clark, D. M. (2000). A cognitive model of posttraumatic stress disorder. Behaviour Research and Therapy, 38(4), 319-345. doi: 10.1016/s00057967(99)00123-0

Elmir, R., Schmied, V., Wilkes, L., \& Jackson, D. (2010). Women's perceptions and experiences of a traumatic birth: a meta-ethnography. Journal of Advanced Nursing, 66(10), 2142-2153. doi: 10.1111/j.1365-2648.2010.05391.x

Elwood, L. S., Mott, J., Lohr, J. M., \& Galovski, T. E. (2011). Secondary trauma symptoms in clinicians: A critical review of the construct, specificity, and implications for trauma-focused treatment. Clinical Psychology Review, 31(1), 25-36. doi: 10.1016/j.cpr.2010.09.004 
Midwives' experiences of traumatic perinatal events

1 Figley, C.R. (1995). Compassion fatigue as secondary traumatic stress disorder: An overview. In C. R. Figley (Ed.), Compassion fatigue: Coping with secondary traumatic stress disorder in those who treat the traumatized (1-20). New York, USA: Routledge.

Foa, E. B., Steketee, G., \& Rothbaum, B. O. (1989). Behavioural/cognitive conceptualisation of post-traumatic stress disorder. Behaviour Therapy, 20, 155- 176.

Goldbort, J., Knepp, A., Mueller, C., \& Pyron, M. (2011). Intrapartum nurses’ lived experience in a traumatic birthing process. $M C N$ - The American Journal of MaternalChild Nursing, 36(6). 373-380 doi: 10.1097/NMC.0b013e31822de535

Health and Social Care Information Centre. (2009). Adult Psychiatric Morbidity in England2007, Results of a household survey. Retrieved from http://www.hscic.gov.uk/catalogue/PUB02931

Health and Social Care Information Centre. (2013). NHS workforce: Summary of staff in the NHS: Results from September 2012 census. Retrieved from http://www.hscic.gov.uk/catalogue/PUB10392

Health Workforce Australia (2012). Health Workforce 2015: Doctors, Nurses and Midwives. Volume 1.Retrived from http://www.hwa.gov.au/sites/uploads/FinalReport_Volume1_FINAL-20120424.pdf

International Confederation of Midwives. (2011). International definition of a midwife. Retrieved from http://www.internationalmidwives.org/who-we-are/policy-andpractice/icm-international-definition-of-the-midwife/

Horowitz, M., Wilner, N., \& Alvarez, W. (1979). Impact of event scale- measure of subjective stress. Psychosomatic Medicine, 41(3), 209-218.

Information Services Division (ISD) Scotland (2014). NHS Scotland workforce statistics; Nursing and midwifery staff by location of service delivery, speciality, age group, NHS region and board. Retrieved from http://www.isdscotland.org/Health- 
Midwives' experiences of traumatic perinatal events

1 Janoff-Bulman, R. (1989). Assumptive worlds and the stress of traumatic events Applications of the schema construct Social Cognition, 7(2), 113-136. doi: 10.1521/soco.1989.7.2.113

Jenkins, S., \& Baird, S. (2002). Secondary traumatic stress and vicarious trauma: A validational study. Journal of Traumatic Stress, 15(5), 423- 432.

Kirkham, M. (2007). Traumatised midwives. AIMS Journal 19(1), 12-13.

Kline, P. (1999). The handbook of psychological testing (2nd ed.). London: Routledge

Leinweber, J., \& Rowe, H. J. (2010). The costs of 'being with the woman': secondary traumatic stress in midwifery. Midwifery, 26(1), 76-87. doi: 10.1016/j.midw.2008.04.003

Leiter, M. P., \& Maslach, C. (2009). Nurse turnover: the mediating role of burnout. Journal of Nursing Management, 17(3), 331-339. doi: 10.1111/j.1365-2834.2009.01004.x

Malott, A. M., Davis, B. M., McDonald, H., \& Hutton, E. (2009). Midwifery care in eight industrialized countries: how does Canadian midwifery compare? Journal of obstetrics and gynaecology Canada, 31(10), 974-979.

Mander, R. (2001). The midwife's ultimate paradox: a UK-based study of the death of a mother. Midwifery, 17(4), 248-258. doi: 10.1054/midw.2001.0275

Maslach, C., Jackson, S. E., Leiter, M.P., Schaufeli, W. B., \& Schwab, R.L. (1996). MBI: The Maslach Burnout Inventory: Manual. Palo Alto: Consulting Psychologists Press.

McCann, I., Pearlman, L. A. (1990). Vicarious traumatization: A framework for understanding the psychological effects of working with victims. Journal of Traumatic Stress, 3(1), 131-149.

Mealer, M., Jones, J., Newman, J., McFann, K. K., Rothbaum, B., \& Moss, M. (2012). The presence of resilience is associated with a healthier psychological profile in intensive care unit (ICU) nurses: Results of a national survey. International Journal of Nursing Studies, 49(3), 292-299. doi: 10.1016/j.ijnurstu.2011.09.015 
Midwives' experiences of traumatic perinatal events

1 Mollart, L., Skinner, V. M., Newing, C., \& Foureur, M. (2013). Factors that may influence midwives work-related stress and burnout. Women and Birth, 26(1), 26-32. doi: 10.1016/j.wombi.2011.08.002

4 5

Nunnally, J.C. \& Bernstein, I.H. (1994). Psychometric Theory. 3rd edition. New York: McGraw-Hill.

Nursing and Midwifery Council. (2008). Statistical analysis of the register 1 April 2007 to 31 March 2008. Retrieved from http://www.nmcuk.org/Documents/Statistical\%20analysis\%20of\%20the\%20register/NMC-Statisticalanalysis-of-the-register-2007-2008.pdf

Olde, E., van der Hart, O., Kleber, R., \& van Son, M. (2006). Posttraumatic stress following childbirth: A review. Clinical Psychology Review, 26(1), 1-16. doi: 10.1016/j.cpr.2005.07.002

Royal College of Midwives. (2013). State of Maternity Services Report 2012. Accessed December 2013, retrieved from http://www.rcm.org.uk/college/policypractice/government-policy/state-of-maternity-services/

Sheen K., Slade P. \& Spiby H. (2014). An integrative review of the impact of indirect trauma exposure in health professionals and potential issues of salience for midwives. Journal of Advanced Nursing, 70(4), 729-743. doi: 10.1111/jan.12274

ten Hoope-Bender, P., de Bernis, L., Campbell, J., Downe, S., Fauveau, V., Fogstad, H., Homer, C, S. E., Kennedy, H. P., Matthews, Z., McFadden, A., Renfrew, M. J. \& Van Lerberghe, W. (2014). Improvement of maternal and newborn health through midwifery. The Lancet.

Thomas, B. G. (2006). 'Making a difference:' midwives’ experiences of caring for women. Evidence Based Midwifery, 4(3), 83-88. 
Midwives' experiences of traumatic perinatal events

1 Wallbank, S. (2010). Effectiveness of individual clinical supervision for midwives and 2 doctors in stress reduction: findings from a pilot study. Evidence Based Midwifery,

3 $8(2), 65-70$.

4 Weiss, D., \& Marmar, C. (1997). The Impact of Event Scale -Revised. In J. Wilson, T. Keane 5 (Eds.), Assessing psychological trauma and PTSD (pp. 399- 411). New York, USA:

6 Guildford

7 
Table 1 Personal characteristics of midwives within the postal survey $(n=421)$

\begin{tabular}{|c|c|c|c|c|}
\hline & $N$ & $M$ & $S D$ & Range \\
\hline Age (years) & 421 & 45.04 & 9.85 & $22-48$ \\
\hline Characteristics & Subcategory & $N$ & $\%$ & \\
\hline \multirow[t]{2}{*}{ Gender } & Female & 420 & 99.8 & \\
\hline & Male & 1 & .2 & \\
\hline \multirow[t]{3}{*}{ Education } & Bachelor's/ RM/ SCM & 264 & 62.7 & \\
\hline & Diploma/ Cert. & 104 & 24.6 & \\
\hline & Master's/ Doctorate & 29 & 6.9 & \\
\hline \multirow[t]{3}{*}{ Marital Status } & Married/ Cohabiting & 328 & 77.9 & \\
\hline & Single & 50 & 11.9 & \\
\hline & Divorced & 35 & 8.3 & \\
\hline \multirow[t]{2}{*}{ Parity } & Nulliparous & 75 & 17.8 & \\
\hline & Multiparous & 334 & 79.4 & \\
\hline \multirow[t]{4}{*}{ NHS Band } & 5 & 9 & 2.2 & \\
\hline & 6 & 272 & 63.6 & \\
\hline & 7 & 108 & 25.4 & \\
\hline & $8 a-d$ & 16 & 5.2 & \\
\hline \multirow[t]{2}{*}{ Clinical practice } & Yes & 395 & 93.8 & \\
\hline & No & 24 & 5.7 & \\
\hline \multirow[t]{4}{*}{ Area of practice* } & Labour ward/ Intrapartum care & 253 & 60.1 & \\
\hline & Community & 146 & 34.7 & \\
\hline & Postnatal & 128 & 30.4 & \\
\hline & Antenatal & 132 & 31.4 & \\
\hline
\end{tabular}

Note $* \%$ not mutually exclusive as midwives reported concurrent role, \% indicates

proportion of total sample $(n=421)$ 
Midwives' experiences of traumatic perinatal events

Table 2 Descriptive statistics for posttraumatic stress symptoms (Impact of Event ScaleRevised), worldview beliefs (World Assumptions Scale) and burnout (Maslach Burnout Inventory)

\begin{tabular}{|c|c|c|c|c|}
\hline Scale & Subscale ( $n$ items) & Range & Mean & $\begin{array}{l}\text { Standard } \\
\text { deviation }\end{array}$ \\
\hline Impact of Event & Intrusion (8) & $0-32$ & 12.74 & 7.65 \\
\hline Scale- Revised & Avoidance (8) & $0-28$ & 8.13 & 6.14 \\
\hline (IES-R) & Arousal (6) & $0-24$ & 6.63 & 5.85 \\
\hline$(n=416)$ & Total (24) & $0-84$ & 27.49 & 17.85 \\
\hline World & Benevolence of the world (8) & $68-161$ & 122.15 & 15.02 \\
\hline Assumptions & Meaningfulness of the world & $13-48$ & 37.65 & 5.61 \\
\hline Scale (WAS) & (12) & & & \\
\hline \multirow[t]{2}{*}{$(n=418)$} & Self-worth (12) & $13-53$ & 34.00 & 7.21 \\
\hline & Total (32) & $23-71$ & 50.50 & 8.59 \\
\hline Maslach Burnout & Emotional Exhaustion (9) & $0-54$ & 23.81 & 11.49 \\
\hline Inventory (MBI) & Depersonalisation (5) & $0-30$ & 3.84 & 4.05 \\
\hline \multirow[t]{8}{*}{$(n=385)$} & Personal Accomplishment (8) & $0-48$ & 38.94 & 5.86 \\
\hline & & High & Moderate & Low level \\
\hline & & level of & level of & of burnout \\
\hline & & burnout & burnout & \\
\hline & Emotional Exhaustion (n, \%) & $156(39.9)$ & $110(28.1)$ & $125(32.0)$ \\
\hline & Depersonalisation (n, \%) & $16(3.8)$ & $61(14.5)$ & $314(74.6)$ \\
\hline & Personal Accomplishment (n, & $43(10.2)$ & $127(30.2)$ & $222(52.7)$ \\
\hline & $\%)$ & & & \\
\hline
\end{tabular}

Note: *denotes $\%$ of sample completing IES-R $(n=416)$ 
Midwives' experiences of traumatic perinatal events

1

Table 3 Bivariate correlations (Pearson's $r$ ) between personal and professional experience variables and posttraumatic stress symptoms (Impact of Event Scale-Revised)

\begin{tabular}{lllll}
\hline & \multicolumn{4}{c}{ Impact of Event Scale-Revised } \\
\cline { 2 - 5 } & Total & Intrusion & Avoidanc & Arousal \\
& & & $\mathrm{e}$ & \\
Experience in the profession & .04 & .03 & .05 & .02 \\
Empathic Concern & $.13^{*}$ & $.18^{* *}$ & .00 & $.16^{* *}$ \\
Number of traumatic perinatal experience(s) & $.18^{* *}$ & $.16^{* *}$ & $.18^{* *}$ & $.16^{* *}$ \\
Personal trauma history & $-.12^{*}$ & $-.11^{*}$ & $-.12^{*}$ & -.09 \\
Personal childbirth trauma history & -.01 & .01 & .01 & -.06 \\
Both types of exposure & $.21 * *$ & $.19^{* *}$ & $.21 * *$ & $.17^{* *}$ \\
\hline
\end{tabular}

Note: $N=319 ; * p<.05 ; * * p<.001$ 
Midwives' experiences of traumatic perinatal events

Table 4 Standard multiple regression for posttraumatic stress symptoms (Impact of Event Scale-Revised) and personal and professional experience variables

$B \quad S E \quad \beta$

Constant 13.16

7.40

Empathic Concern .37 $.23 \quad .08$

Number of traumatic perinatal $\begin{array}{lll}.08 & .03 \quad .12\end{array}$

experiences

Personal trauma history $-.25$

Both types of exposure

$5.93 \quad 2.02$
.15

Note. $N=402 ; R^{2}=.07 ;$ Adjusted $R^{2}=.06 ; * p<.05 ; * * p<.001$ 
Table 5 Bivariate correlations between burnout (MBI) posttraumatic stress symptoms (IES-R) and worldview beliefs (WAS)

\begin{tabular}{lllllllll}
\hline \multicolumn{4}{c}{ Impact of Event Scale-Revised $(n=387)$} & \multicolumn{5}{c}{ World assumptions Scale $(n=388)$} \\
\cline { 2 - 9 } & INT & AV & AR & Total & BW & MW & SW & Total \\
\hline EE & $.38^{* *}$ & $.38^{* *}$ & $.40^{* *}$ & $.42^{* *}$ & $-.23^{* *}$ & -.08 & $-.32^{* *}$ & $-.31^{* *}$ \\
DP & $.20^{* *}$ & $.26^{* *}$ & $.22^{* *}$ & $.25^{* *}$ & $-.29 * *$ & -.02 & $-.25^{* *}$ & $-.26^{* *}$ \\
PA & .03 & -.06 & -.03 & -.02 & $.25^{* *}$ & -.01 & $.30^{* *}$ & $.26^{* *}$ \\
\hline
\end{tabular}

Note. $A R=$ Arousal $(I E S-R) ; A V=$ Avoidance $(I E S-R) ; B W=$ Benevolence of the World $(W A S) ; E E=$ Emotional Exhaustion $(M B I), D P=$ Depersonalisation $(M B I), I N T=$ Intrusion (IES-R); MW= Meaningfulness of the World (WAS); PA= Personal Accomplishment (MBI); SW=Self-worth (WAS) $* p<.05 ; * * p<.001$ 
Table 6 Standard multiple regression analyses for subscales from the Maslach Burnout Inventory (MBI) and subscale scores on the Impact of Event Scale- Revised (IES-R) and the World Assumptions Scale (WAS)

\section{Maslach Burnout Inventory (MBI)}

\section{Emotional Depersonalisation Perceived}

Exhaustion

Accomplishment

\begin{tabular}{|c|c|c|c|c|c|c|c|c|c|}
\hline & $B$ & $S E$ & $\beta$ & $B$ & $S E$ & $\beta$ & $B$ & $S E$ & $\beta$ \\
\hline Constant & 39.42 & 4.23 & & 11.31 & 1.59 & & 24.68 & 2.15 & \\
\hline Intrusion (IES-R) & .14 & .13 & .10 & .02 & .05 & .05 & - & - & - \\
\hline Avoidance (IES-R) & $.32 *$ & .12 & .17 & $.12 *$ & .05 & .19 & - & - & - \\
\hline Arousal (IES-R) & .26 & .18 & .13 & -.01 & .07 & -.01 & - & - & 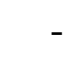 \\
\hline Benevolence of the & $-.20 *$ & .10 & -.10 & $.16^{* *}$ & .04 & -.22 & $.16^{*}$ & .6 & .16 \\
\hline \multicolumn{10}{|l|}{ world (WAS) } \\
\hline Self-worth (WAS) & $-.279 * *$ & .07 & -.21 & $.06^{*}$ & .03 & -.12 & $.16 * *$ & .04 & .24 \\
\hline \multicolumn{10}{|c|}{ Note. $N=384 ; E E: R^{2}=.24 ;$ Adjusted $R^{2}=.23 ; D P: R^{2}=.15 ;$ Adjusted $R^{2}=.14 ; P A: R^{2}=$} \\
\hline
\end{tabular}

